Supplement of Biogeosciences, 16, 3941-3958, 2019

https://doi.org/10.5194/bg-16-3941-2019-supplement

(C) Author(s) 2019. This work is distributed under

the Creative Commons Attribution 4.0 License.

(c) (1)

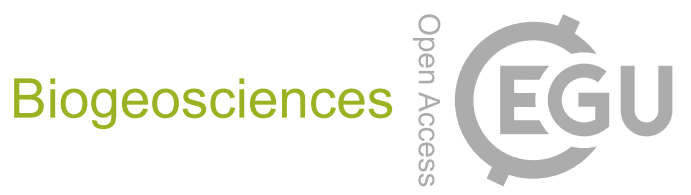

Supplement of

\title{
Microbial community composition and abundance after millennia of submarine permafrost warming
}

Julia Mitzscherling et al.

Correspondence to: Susanne Liebner (susanne.liebner@gfz-potsdam.de)

The copyright of individual parts of the supplement might differ from the CC BY 4.0 License. 


\section{Supplementary Online Material:}

\section{Contents:}

Figures S1-S5: including DNA concentrations, DOC contents and additional information to Figure 2 (PCA) as well as a schematic description of the formation of the sediments units.

Tables S1-S14: including a site description, an overview of selected physicochemical parameters and of the microbial abundances, detailed information about the molecular samples, sequencing primers and barcode sequences, sequencing statistics as well as the results of all statistical tests 


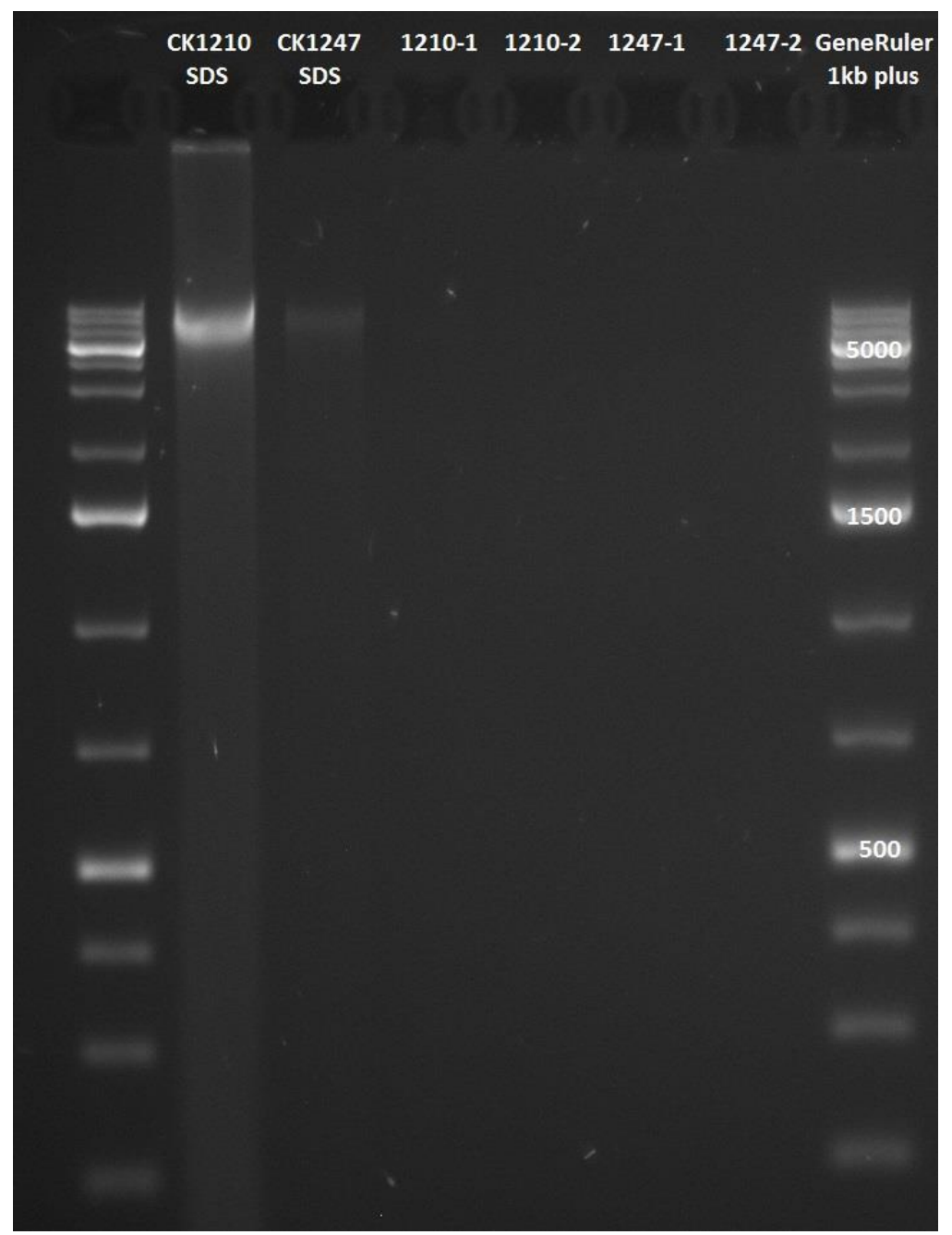

Figure S1: Quality control of the extracted genomic DNA, exemplarily shown for two samples from core C2 (CK1210 SDS: $0.05 \mathrm{~m}$ bsf, $265 \mathrm{ng} / \mathrm{g}$ and CK1247 SDS: $52.7 \mathrm{~m}$ bsf, $33.4 \mathrm{ng} / \mathrm{g}$ ). The examples show that there is not much fragmentation likely due to constantly freeze-locked conditions. Hence, we used the DNA extracts without gel purification for downstream analyses. 


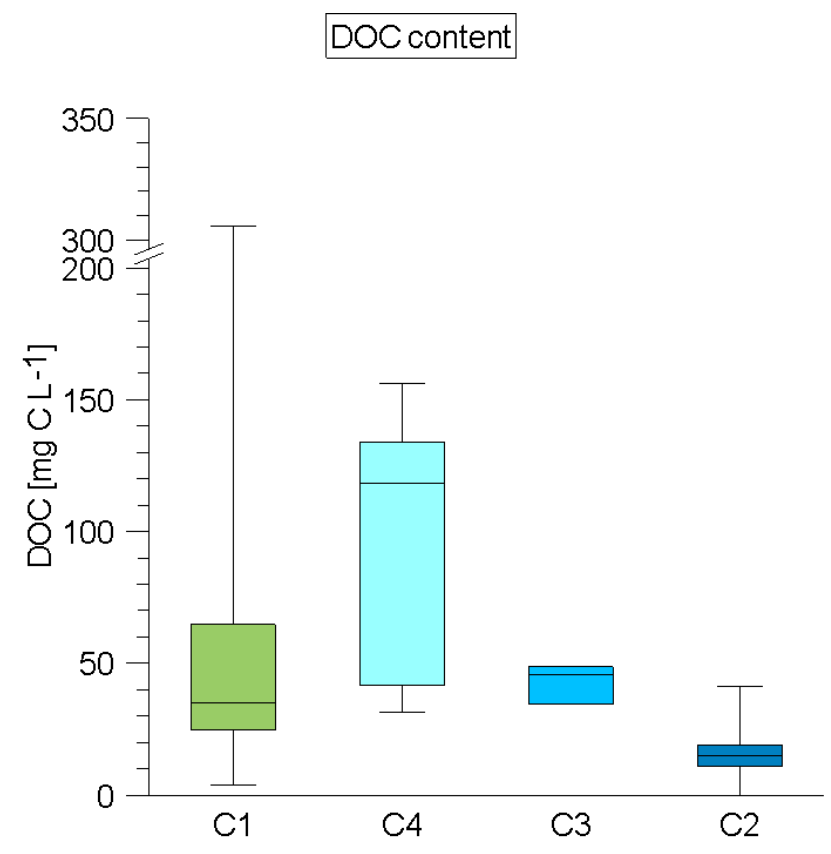

Figure S2: Boxplot of DOC concentrations of Unit II in mg C L-1. Median lines are indicated within the boxes of which the size corresponds to $\pm 25 \%$ of the data, whereas the whiskers show the minimum and maximum of all data. $\mathrm{C} 1: \mathrm{n}=74, \mathrm{C} 4: \mathrm{n}=5, \mathrm{C} 3: \mathrm{n}=3, \mathrm{C} 2: \mathrm{n}=12$.

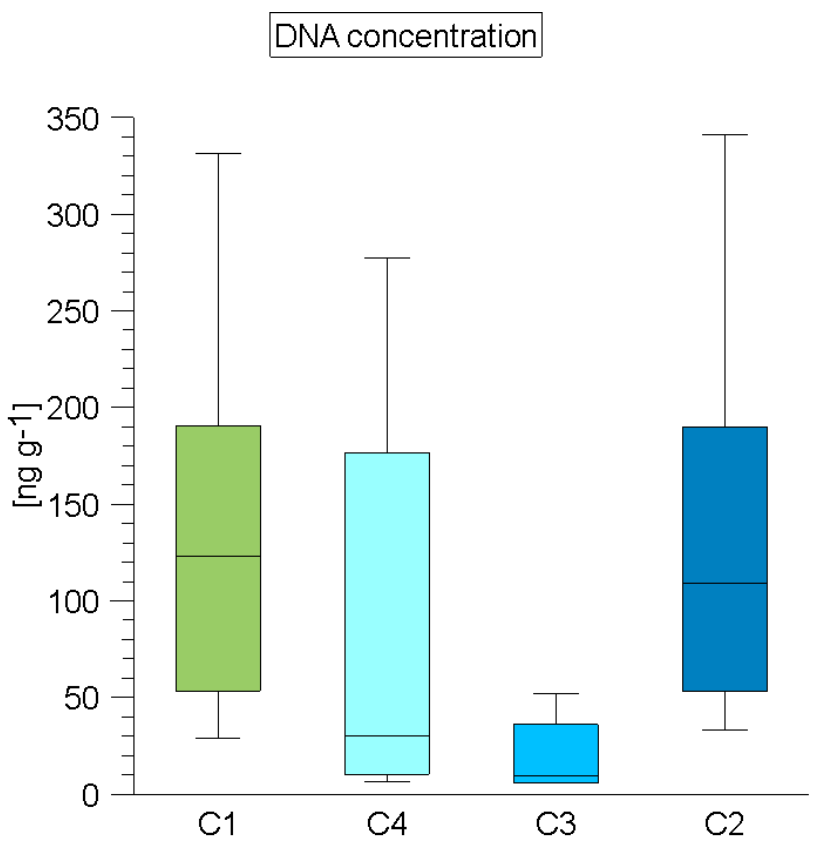

Figure S3: DNA concentrations in $\mathrm{ng} \mathrm{g}^{-1}$ sediment wet weight of the cores $\mathrm{C} 1, \mathrm{C} 4, \mathrm{C} 3$ and C2. Box plots contain the mean values of all samples, obtained from two technical replicates each. Median lines are indicated within the boxes of which the size corresponds to $\pm 25 \%$ of 
the data, whereas the whiskers show the minimum and maximum of all data. $\mathrm{C} 1, \mathrm{C} 4$ and $\mathrm{C} 3$ : $\mathrm{n}=6, \mathrm{C} 2: \mathrm{n}=17$.
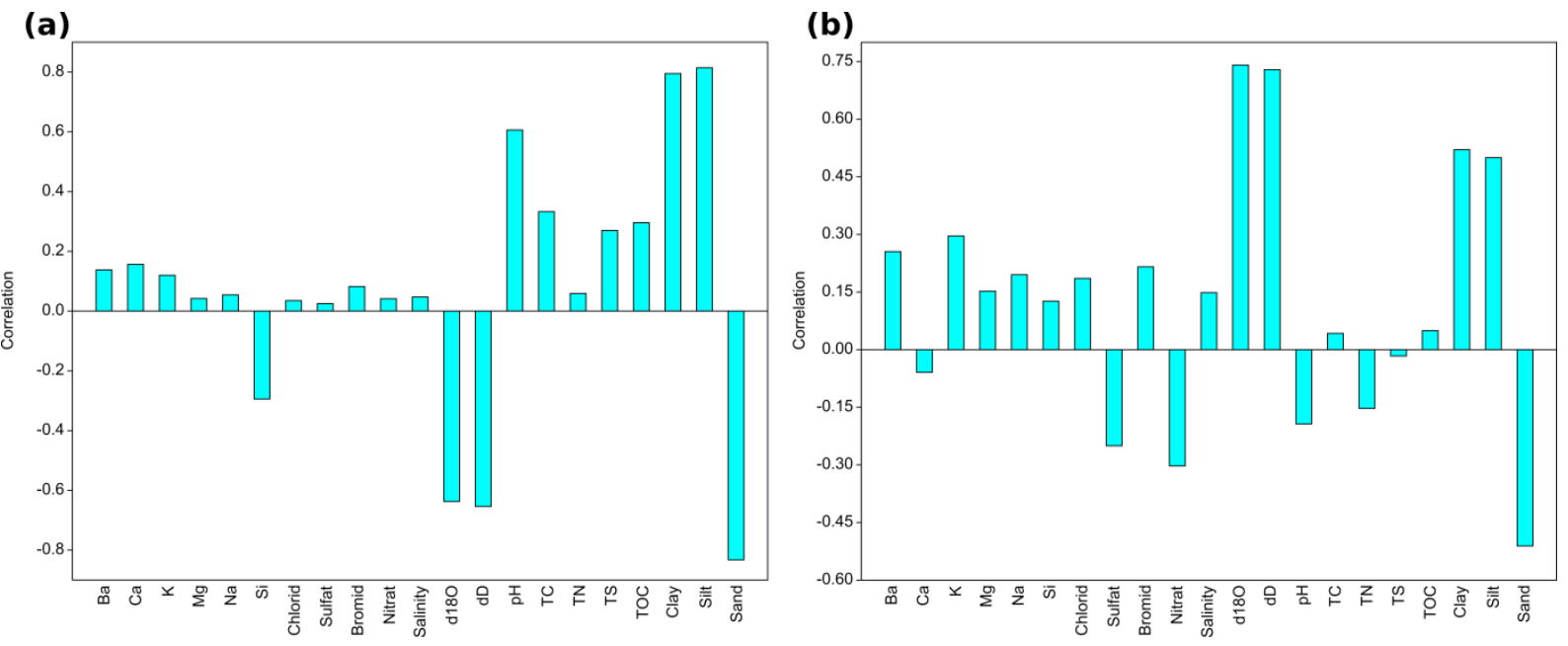

Figure S4: Loadings plots belonging to Figure 3: PCA of environmental, sedimentological and pore water data from Unit II. Shown are the correlations to a) PC1 and b) PC2 which were used to choose the physicochemical factors that are mainly responsible for the variance between samples in the PCA. 


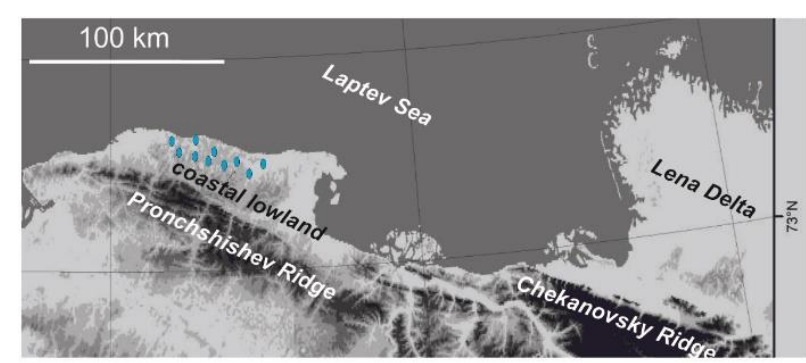

D. modern coastal lowland of the western Laptev Sea

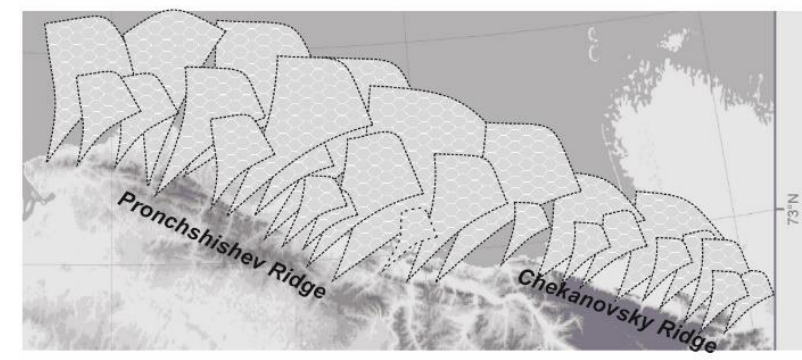

C. supposed Late Weichselian flood plain area of Ice Complex accumulation

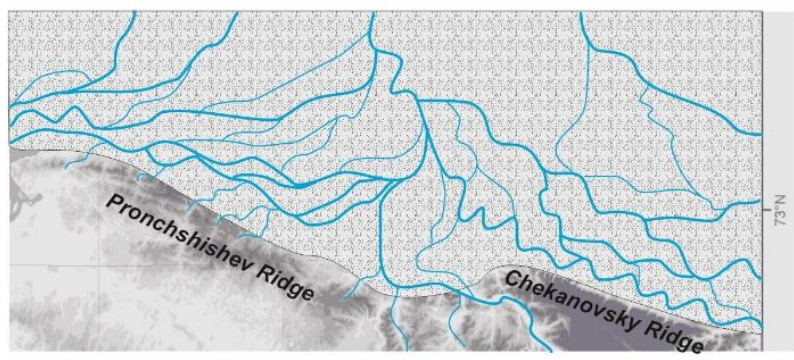

B. supposed Early to Middle Weichselian fluvial dominated terrestrial shelf

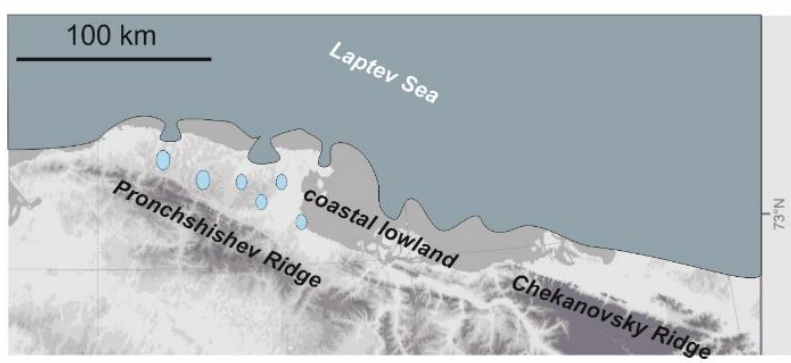

A. supposed Eemian coastal lowland with brackish thermokarstlagoons and terrestrial thermokarst bassins
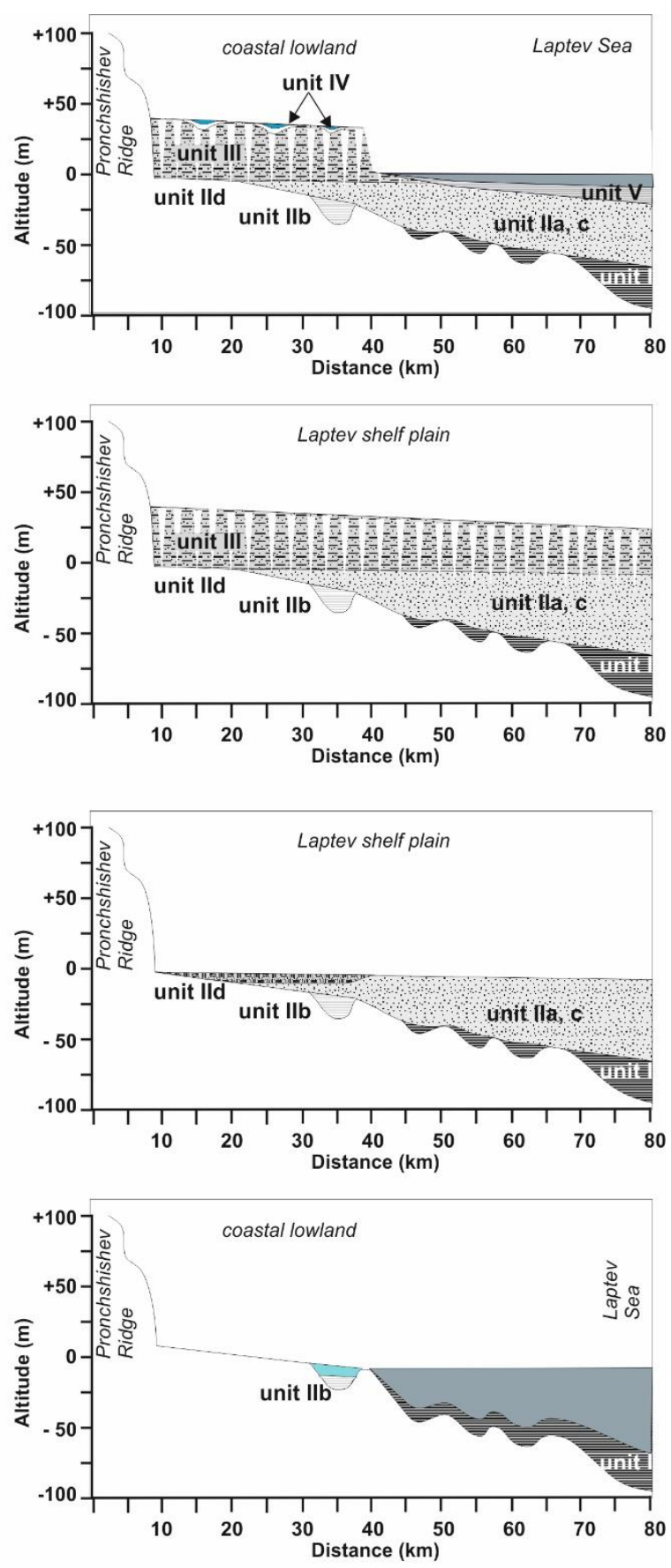

River branches of a braided river system

Thermokarst lake

Figure S5: Schematic representation of the late Quaternary landscape dynamics in the western Laptev Sea coastal region and formation of the sediments units (modified after Winterfeld et al. (2011)). 
Table S1: Site description of each borehole location. Mbsf stands for meters below sea floor and dedicates the depth in the submarine cores $(\mathrm{C} 4, \mathrm{C} 3, \mathrm{C} 2)$, while mbs stands for meters below surface and is used for depth indication in the terrestrial core $\mathrm{C} 1$.

\begin{tabular}{l|c|c|c|c} 
& C1 & C4 & C3 & C2 \\
\hline Distance to coast [km] & -0.1 & 1.0 & 3.0 & 11.5 \\
\hline Water depth [m] & - & 2.2 & 4.4 & 6.0 \\
\hline Frost table depth [mbsf] & 0.0 & 1.7 & 7.6 & 29.0 \\
\hline Upper boundary of Unit II [mbs/mbsf] & 22.0 & 13.3 & 8.6 & 35.0 \\
Lower boundary of Unit II & 61.0 & 29.8 & 27.1 & 58.5 \\
\hline Uppermost sample depth [mbs/mbsf] & 27.3 & 13.3 & 8.6 & 38.5 \\
Lowermost sample depth & 44.3 & 29.8 & 24.9 & 58.4 \\
\hline
\end{tabular}

Table S2: Minimum, maximum and mean values of environmental factors in Unit II significantly contributing to the bacterial community composition and microbial abundance.

\begin{tabular}{|c|c|c|c|c|c|c|}
\hline & Core & Minimum & Maximum & Mean & Std.Dev. & $\mathrm{n}$ \\
\hline \multirow{4}{*}{$\begin{array}{c}\text { Temperature } \\
{\left[{ }^{\circ} \mathrm{C}\right]}\end{array}$} & $\mathrm{C} 1$ & -12.5 & -12.4 & -12.4 & 0.0 & 8 \\
\hline & $\mathrm{C} 4$ & -7.1 & -5.8 & -6.4 & 0.5 & 4 \\
\hline & C3 & -1.8 & -1.2 & -1.4 & 0.2 & 4 \\
\hline & $\mathrm{C} 2$ & -1.6 & -1.5 & -1.5 & 0.0 & 4 \\
\hline \multirow{4}{*}{ Salinity [PSU] } & C1 & 0.0 & 1.6 & 0.5 & 0.4 & 184 \\
\hline & C4 & 0.9 & 17.6 & 5.6 & 4.8 & 10 \\
\hline & $\mathrm{C} 3$ & 0.5 & 3.7 & 1.0 & 0.6 & 38 \\
\hline & $\mathrm{C} 2$ & 0.0 & 12.5 & 0.8 & 1.7 & 67 \\
\hline \multirow{4}{*}{$\begin{array}{c}\delta 180 \\
{[(\% \circ) \text { vs. }} \\
\text { SMOW] }\end{array}$} & $\mathrm{C} 1$ & -30.8 & -14.9 & -22.3 & 4.0 & 184 \\
\hline & $\mathrm{C} 4$ & -27.7 & -18.8 & -22.8 & 3.0 & 10 \\
\hline & C3 & -20.6 & -19.1 & -20.1 & 0.3 & 38 \\
\hline & $\mathrm{C} 2$ & -30.0 & 20.2 & -27.6 & 1.5 & 67 \\
\hline \multirow{4}{*}{$\begin{array}{c}\delta D \\
{[(\% \circ) \text { vs. }} \\
\text { SMOW] }\end{array}$} & C1 & -241.8 & -115.7 & -177.2 & 32.9 & 184 \\
\hline & C4 & -219.1 & -144.0 & -178.8 & 25.7 & 10 \\
\hline & $\mathrm{C} 3$ & -162.9 & -149.4 & -158.4 & 2.9 & 38 \\
\hline & $\mathrm{C} 2$ & -232.7 & -156.8 & -213.3 & 11.3 & 67 \\
\hline
\end{tabular}

Table S3: Geochemical, pore water and environmental data of all samples at each drill site.

See "SuppInfo_Table_3" 
Table S 4: Sample names of the molecular samples, their depth relative to sea level (meters below sea level, $\mathrm{m}$ bsl), relative to surface (meters below surface ( $\mathrm{m} \mathrm{bs}$ ) in the terrestrial core and meters below sea floor ( $\mathrm{m} \mathrm{bsf}$ ) in the submarine cores), and their corresponding lithology.

\begin{tabular}{|c|c|c|c|}
\hline Sample Name & $\begin{array}{l}\text { Depth [m } \\
\text { bsl] }\end{array}$ & $\begin{array}{l}\text { Depth [m bs/ m } \\
\text { bsf] }\end{array}$ & Lithology \\
\hline C1-1 & 1.3 & 27.3 & sandy \\
\hline C1-2 & 9.9 & 35.9 & sandy \\
\hline C1-3 & 12.1 & 38.1 & sandy \\
\hline C1-4 & 17.2 & 43.2 & plant/wood detritus \\
\hline C1-5 & 17.4 & 43.4 & plant/wood detritus \\
\hline C1-6 & 18.4 & 44.4 & plant/wood detritus \\
\hline C4-1 & 15.5 & 13.3 & plant/wood detritus \\
\hline C4-2 & 22.0 & 19.8 & small peat inclusions \\
\hline C4-3 & 25.0 & 22.8 & small peat inclusions \\
\hline C4-4 & 28.5 & 26.3 & sandy with quartz gravel \\
\hline C4-5 & 30.0 & 27.8 & sandy with quartz gravel \\
\hline C4-6 & 32.0 & 29.8 & sandy with quartz gravel \\
\hline C3-1 & 13.0 & 8.6 & small peat inclusions \\
\hline C3-2 & 16.0 & 11.6 & small peat inclusions \\
\hline C3-3 & 19.0 & 14.6 & small peat inclusions \\
\hline C3-4 & 21.5 & 17.1 & sandy \\
\hline C3-5 & 24.6 & 20.2 & sandy \\
\hline C3-6 & 29.2 & 24.8 & sandy \\
\hline CK1232 & 44.5 & 38.5 & \\
\hline C2-1 & 46.0 & 40.0 & sandy \\
\hline C2-2 & 48.1 & 42.1 & sandy \\
\hline CK1235 & 49.3 & 43.3 & \\
\hline CK1236 & 50.7 & 44.7 & \\
\hline CK1237 & 51.8 & 45.8 & \\
\hline C2-4 & 52.2 & 46.2 & sandy \\
\hline C2-5 & 54.6 & 48.6 & plant/wood detritus \\
\hline CK 1241 & 54.7 & 48.7 & \\
\hline C2-7 & 55.0 & 49.0 & plant/wood detritus \\
\hline C2-8/1244 & 56.1 & 50.1 & plant/wood detritus \\
\hline CK1245 & 57.8 & 51.8 & \\
\hline CK1246 & 58.2 & 52.2 & \\
\hline CK1247 & 58.7 & 52.7 & \\
\hline CK1248 & 61.6 & 55.6 & \\
\hline C2-9 & 62.9 & 56.9 & plant/wood detritus \\
\hline C2-10 & 64.4 & 58.4 & sandy silt \\
\hline
\end{tabular}


Table S5: Oligonucleotide primers for Illumina MiSeq sequencing and quantitative PCR.

\begin{tabular}{|c|c|c|c|c|c|c|}
\hline Target & Primer Sets & Primer Sequence 5'-3' & Size bp & $\mathbf{T}\left({ }^{\circ} \mathbf{C}\right)$ & $\begin{array}{c}\text { No. of PCR } \\
\text { Cycles }\end{array}$ & References \\
\hline \multicolumn{7}{|c|}{ Illumina MiSeq sequencing } \\
\hline Bacterial $16 \mathrm{~S}$ rRNA & $\begin{array}{l}\text { S-D-Bact-0341-b- } \\
\text { S-17 } \\
\text { S-D-Bact-0785-a- } \\
\text { A-21 }\end{array}$ & $\begin{array}{c}\text { CCT ACG GGA GGC AGC AG } \\
\text { GAC TAC HVG GGT ATC TAA TCC }\end{array}$ & 464 & 55 & 35 & $\begin{array}{l}\text { (Muyzer et al., } \\
\text { 1993) } \\
\text { (Herlemann et } \\
\text { al., 2011) }\end{array}$ \\
\hline \multicolumn{7}{|c|}{ Quantitative PCR } \\
\hline Bacterial $16 S$ rRNA & $\begin{array}{l}\text { S-D-Bact-0341-b- } \\
\text { S-17 } \\
\text { S-D-Bact-0517-a- } \\
\text { A-18 }\end{array}$ & $\begin{array}{l}\text { CCT ACG GGA GGC AGC AG } \\
\text { ATT ACC GCG GCT GCT GG }\end{array}$ & 193 & 55.7 & 40 & $\begin{array}{l}\text { (Muyzer et al., } \\
1993) \\
\text { (Muyzer et al., } \\
1993)\end{array}$ \\
\hline
\end{tabular}

Table S6: Barcode sequences for Illumina MiSeq sequencing.

\begin{tabular}{|c|c|c|c|}
\hline $\begin{array}{c}\text { Barcode ID } \\
\text { Forward Primer }\end{array}$ & Barcode Sequence & $\begin{array}{c}\text { Barcode ID } \\
\text { Reverse Primer }\end{array}$ & Barcode Sequence \\
\hline Bac-01-For & ACGAGTGCGT & Bac-01-Rev & ACGAGTGCGT \\
\hline Bac-02-For & ACGCTCGACA & Bac-02-Rev & ACGCTCGACA \\
\hline Bac-03-For & AGACGCACT & Bac-04-Rev & AGCACTGTAG \\
\hline Bac-06-For & ATATCGCGAG & Bac-05-Rev & ATCAGACACG \\
\hline Bac-07-For & CGTGTCTCTA & Bac-06-Rev & ATATCGCGAG \\
\hline Bac-08-For & CTCGCGTGT & Bac-07-Rev & CGTGTCTCTA \\
\hline Bac-11-For & TGATACGTCT & Bac-08-Rev & CTCGCGTGTC \\
\hline Bac-13-For & CATAGTAGTG & Bac-11-Rev & TGATACGTCT \\
\hline Bac-15-For & ATACGACGTA & Bac-13-Rev & CATAGTAGTG \\
\hline Bac-16-For & TCACGTACTA & Bac-14-Rev & CGAGAGATAC \\
\hline Bac-17-For & CGTCTAGTA & Bac-17-Rev & CGTCTAGTAC \\
\hline Bac-19-For & TGTACTACT & Bac-18-Rev & TCTACGTAGC \\
\hline Bac-23-For & TACTCTCGTG & Bac-19-Rev & TGTACTACTC \\
\hline Bac-24-For & TAGAGACGAG & Bac-22-Rev & TACGAGTATG \\
\hline Bac-25-For & TCGTCGCTCG & Bac-23-Rev & TACTCTCGTG \\
\hline Bac-26-For & ACATACGCGT & Bac-24-Rev & TAGAGACGAG \\
\hline Bac-27-For & ACGCGAGTAT & Bac-25-Rev & TCGTCGCTCG \\
\hline Bac-28-For & АCTACTATGT & Bac-26-Rev & ACATACGCGT \\
\hline Bac-31-For & AGCGTCGTCT & Bac-28-Rev & ACTACTATGT \\
\hline Bac-33-For & ATAGAGTACT & Bac-30-Rev & AGACTATACT \\
\hline Bac-34-For & CACGCTACGT & Bac-31-Rev & AGCGTCGTCT \\
\hline Bac-35-For & CAGTAGACGT & Bac-33-Rev & ATAGAGTACT \\
\hline Bac-36-For & CGACGTGACT & Bac-34-Rev & CACGCTACGT \\
\hline Bac-38-For & TACACGTGAT & Bac-35-Rev & CAGTAGACGT \\
\hline Bac-39-For & TACAGATCGT & Bac-36-Rev & CGACGTGACT \\
\hline Bac-40-For & TACGCTGTCT & Bac-37-Rev & TACACACACT \\
\hline Bac-41-For & TAGTGTAGAT & Bac-38-Rev & TACACGTGAT \\
\hline Bac-42-For & TCGATCACGT & Bac-39-Rev & TACAGATCGT \\
\hline
\end{tabular}




\begin{tabular}{l|l|l|l} 
Bac-44-For & TCTAGCGACT & Bac-40-Rev & TACGCTGTCT \\
Bac-45-For & TCTATACTAT & Bac-41-Rev & TAGTGTAGAT \\
Bac-49-For & ACGCGATCGA & Bac-44-Rev & TCTAGCGACT \\
Bac-50-For & ACTAGCAGTA & Bac-45-Rev & TCTATACTAT \\
& & Bac-46-Rev & TGACGTATGT \\
& & Bac-49-Rev & ACGCGATCGA \\
\hline SfiA-MW00 & ACACGT & SfiB-MW10 & CAGTCA \\
SfiA-MW01 & ACGTAC & SfiB-MW11 & CATGAC \\
SfiA-MW02 & ACTGCA & SfiB-MW12 & GACTAG \\
SfiA-MW02 & ACTGCA & SfiB-MW13 & GAGATC \\
SfiA-MW03 & AGAGTC & SfiB-MW14 & GATCGA \\
SfiA-MW04 & AGCTGA & SfiB-MW14 & GATCGA \\
SfiA-MW05 & AGTCAG & SfiB-MW15 & GTACAC \\
SfiA-MW06 & ATATCG & SfiB-MW15 & GTACAC \\
SfiA-MW07 & ATCGAT & SfiB-MW16 & GTCACA \\
& & SfiB-MW17 & GTGTGT \\
\hline
\end{tabular}

Table S 7: Overview of sequencing reads: number of reads after the removal of singletons, number of reads that were removed when the background filter of $0.5 \%$ was applied, number of reads representing chloroplast, mitochondrial and archaeal taxa and finally the number of quality reads after the application of all filters. Critical samples with less than 15.000 raw reads are shaded red. Critical samples where the relative abundances within duplicates are comparable are colored light red. The dark red colored sample was not used for the calculation of the mean relative abundance as the relative abundances within duplicates differed.

\begin{tabular}{l|rrrrrr} 
& Raw reads & $\begin{array}{c}\text { Background } \\
\text { Reads (0.5\%) }\end{array}$ & Chloroplast & $\begin{array}{c}\text { Mitochondrial } \\
\text { Taxa }\end{array}$ & Archaeal taxa & Quality reads \\
\hline C1-1a & 74760 & 20214 & 0 & 0 & 0 & 54546 \\
C1-1b & 89992 & 22879 & 0 & 0 & 0 & 67113 \\
C1-2a & 23789 & 15150 & 0 & 0 & 0 & 8639 \\
C1-2b & 25100 & 16330 & 0 & 0 & 0 & 8770 \\
C1-3a & 41727 & 24707 & 0 & 0 & 0 & 17020 \\
C1-3b & 8666 & 5185 & 0 & 0 & 0 & 3481 \\
C1-4a & 31071 & 22620 & 0 & 0 & 0 & 8451 \\
C1-4b & 5142 & 3761 & 0 & 0 & 0 & 1381 \\
C1-5a & 208578 & 100128 & 0 & 0 & 0 & 108450 \\
C1-5b & 147753 & 69180 & 0 & 0 & 0 & 78573 \\
C1-6a & 244866 & 100302 & 0 & 0 & 1790 & 142774 \\
C1-6b & 255535 & 113256 & 0 & 0 & 6331 & 135948 \\
\hline C4-1a & 231425 & 70205 & 0 & 0 & 0 & 161220 \\
C4-1b & 103692 & 30996 & 0 & 0 & 0 & 72696 \\
C4-2a & 312930 & 64767 & 0 & 0 & 0 & 248163 \\
C4-2b & 18603 & 2840 & 0 & 0 & 0 & 15763 \\
C4-3a & 269853 & 99103 & 1765 & 0 & 0 & 168985 \\
C4-3b & 94463 & 33930 & 0 & 0 & 0 & 60533
\end{tabular}




\begin{tabular}{|c|c|c|c|c|c|c|}
\hline C4-4a & 170018 & 49851 & 0 & 0 & 1050 & 119117 \\
\hline$C 4-4 b$ & 180556 & 52147 & 0 & 0 & 0 & 128409 \\
\hline C4-5a & 9823 & 4402 & 0 & 0 & 0 & 5421 \\
\hline$C 4-5 b$ & 17374 & 7566 & 0 & 0 & 0 & 9808 \\
\hline C4-6a & 56201 & 23871 & 0 & 0 & 0 & 32330 \\
\hline C4-6b & 20949 & 8951 & 0 & 0 & 0 & 11998 \\
\hline C3-1a & 52885 & 27176 & 0 & 0 & 588 & 25121 \\
\hline$C 3-1 b$ & 180772 & 94154 & 1165 & 0 & 2402 & 83051 \\
\hline C3-2a & 35528 & 13955 & 0 & 0 & 1511 & 20062 \\
\hline$C 3-2 b$ & 102122 & 39090 & 652 & 0 & 4780 & 57600 \\
\hline C3-3a & 73935 & 22562 & 0 & 0 & 0 & 51373 \\
\hline C3-3b & 25589 & 7940 & 0 & 0 & 0 & 17649 \\
\hline C3-4a & 53553 & 16543 & 0 & 0 & 1899 & 35111 \\
\hline C3-4b & 22552 & 6552 & 0 & 0 & 398 & 15602 \\
\hline C3-5a & 128366 & 34045 & 1091 & 0 & 0 & 93230 \\
\hline$C 3-5 b$ & 16643 & 4179 & 152 & 0 & 0 & 12312 \\
\hline C3-6a & 80004 & 20997 & 0 & 0 & 1349 & 57658 \\
\hline C3-6b & 89902 & 21079 & 867 & 0 & 0 & 67956 \\
\hline CK1232-1 & 127284 & 59052 & 0 & 0 & 0 & 68232 \\
\hline CK1232-2 & 161752 & 70043 & 0 & 0 & 0 & 91709 \\
\hline C2-1a & 53571 & 16678 & 0 & 0 & 0 & 36893 \\
\hline$C 2-1 b$ & 25615 & 7060 & 0 & 0 & 0 & 18555 \\
\hline C2-2a & 55698 & 20602 & 0 & 0 & 0 & 35096 \\
\hline$C 2-2 b$ & 84301 & 25809 & 0 & 0 & 0 & 58492 \\
\hline CK1235-1 & 206215 & 122462 & 11152 & 0 & 0 & 72601 \\
\hline CK1235-2 & 74429 & 43777 & 4130 & 0 & 0 & 26522 \\
\hline CK1236-1 & 20564 & 11265 & 0 & 0 & 0 & 9299 \\
\hline CK1236-2 & 55725 & 30832 & 0 & 0 & 0 & 24893 \\
\hline CK1237-1 & 102376 & 62617 & 0 & 0 & 0 & 39759 \\
\hline CK1237-2 & 139700 & 86444 & 0 & 0 & 0 & 53256 \\
\hline $\mathrm{C} 2-4 \mathrm{a}$ & 136761 & 74460 & 0 & 0 & 0 & 62301 \\
\hline$c 2-4 b$ & 216318 & 118201 & 0 & 0 & 0 & 98117 \\
\hline$C 2-5 a$ & 48354 & 27604 & 0 & 0 & 0 & 20750 \\
\hline$c 2-5 b$ & 92506 & 55950 & 0 & 0 & 0 & 36556 \\
\hline CK1241-1 & 177526 & 115666 & 0 & 0 & 0 & 61860 \\
\hline CK1241-2 & 142667 & 88091 & 0 & 0 & 0 & 54576 \\
\hline C2-7a & 63745 & 36419 & 0 & 0 & 0 & 27326 \\
\hline$C 2-7 b$ & 159960 & 88818 & 0 & 0 & 0 & 71142 \\
\hline C2-8a & 22420 & 14376 & 0 & 0 & 0 & 8044 \\
\hline $\mathrm{C} 2-8 \mathrm{~b}$ & 130842 & 85938 & 0 & 0 & 0 & 44904 \\
\hline CK1244-1 & 99934 & 54354 & 0 & 0 & 0 & 45580 \\
\hline CK1244-2 & 15808 & 9077 & 0 & 0 & 0 & 6731 \\
\hline CK1245-1 & 81822 & 42330 & 0 & 0 & 0 & 39492 \\
\hline CK1245-2 & 49130 & 24254 & 0 & 0 & 0 & 24876 \\
\hline CK1246-1 & 52169 & 30142 & 0 & 0 & 0 & 22027 \\
\hline CK1246-2 & 70027 & 43178 & 0 & 0 & 0 & 26849 \\
\hline CK1247-1 & 32592 & 14991 & 0 & 0 & 0 & 17601 \\
\hline
\end{tabular}




\begin{tabular}{l|rrrrrr} 
CK1247-2 & 21821 & 9398 & 0 & 0 & 0 & 12423 \\
CK1248-1 & 25455 & 16365 & 0 & 0 & 0 & 9090 \\
CK1248-2 & 48980 & 32070 & 0 & 0 & 0 & 16910 \\
C2-9a & 37303 & 24313 & 0 & 0 & 0 & 12990 \\
C2-9b & 43272 & 26410 & 0 & 0 & 0 & 16862 \\
C2-10a & 1889 & 1288 & 0 & 0 & 0 & 601 \\
C2-10b & 213822 & 155149 & 1128 & 0 & 0 & 57545 \\
\hline
\end{tabular}

Table S 8: Spearman correlation of DNA concentration, bacterial 16S rRNA gene abundance and total cell counts. P-values are shown above the diagonal and the correlation coefficient $r_{s}$ below.

\begin{tabular}{|c|c|c|c|}
\hline & DNA & $\begin{array}{c}\text { 16S rRNA } \\
\text { gene } \\
\text { copies }\end{array}$ & TCC \\
\hline DNA & & $>0.0001$ & $>0.0001$ \\
\hline 16S rRNA gene copies & 0.87 & & 0.0001 \\
\hline TCC & 0.68 & 0.61 & \\
\hline
\end{tabular}

Table S9: Minimum. maximum. mean values and standard deviation of microbial and bacterial abundance. $\mathrm{n}$ indicates the number of samples.

\begin{tabular}{cc|ccccc} 
& Core & Min & Max & Mean & Std. dev. & $\mathbf{n}$ \\
\hline DNA concentration & $\mathrm{C} 1$ & 28.6 & 331.3 & 141.6 & 105.6 & 6 \\
[ng g ${ }^{-1}$ ] & $\mathrm{C} 4$ & 6.2 & 277.5 & 88.5 & 102.6 & 6 \\
& $\mathrm{C} 3$ & 5.6 & 51.9 & 19.8 & 17.8 & 6 \\
& $\mathrm{C} 2$ & 8.7 & 341.5 & 106.9 & 94.0 & 17 \\
\hline 16S rRNA gene copies & $\mathrm{C} 1$ & $2.4 \mathrm{E}+07$ & $4.3 \mathrm{E}+08$ & $1.6 \mathrm{E}+08$ & $1.4 \mathrm{E}+08$ & 6 \\
[g $^{-1}$ sediment] & $\mathrm{C} 4$ & $1.7 \mathrm{E}+06$ & $1.6 \mathrm{E}+08$ & $3.6 \mathrm{E}+07$ & $5.8 \mathrm{E}+07$ & 6 \\
& $\mathrm{C} 3$ & $4.1 \mathrm{E}+06$ & $6.2 \mathrm{E}+07$ & $1.7 \mathrm{E}+07$ & $2.1 \mathrm{E}+07$ & 6 \\
& $\mathrm{C} 2$ & $5.4 \mathrm{E}+06$ & $1.5 \mathrm{E}+09$ & $2.9 \mathrm{E}+08$ & $4.0 \mathrm{E}+08$ & 17 \\
\hline 16S rRNA gene copies & $\mathrm{C} 1$ & $7.6 \mathrm{E}+05$ & $1.4 \mathrm{E}+06$ & $1.0 \mathrm{E}+06$ & $2.6 \mathrm{E}+05$ & 6 \\
[ng ${ }^{-1}$ DNA] & $\mathrm{C} 4$ & $5.8 \mathrm{E}+04$ & $5.8 \mathrm{E}+05$ & $2.7 \mathrm{E}+05$ & $1.6 \mathrm{E}+05$ & 6 \\
& $\mathrm{C} 3$ & $4.9 \mathrm{E}+05$ & $1.2 \mathrm{E}+06$ & $7.6 \mathrm{E}+05$ & $2.2 \mathrm{E}+05$ & 6 \\
& $\mathrm{C} 2$ & $2.6 \mathrm{E}+05$ & $1.7 \mathrm{E}+07$ & $2.7 \mathrm{E}+06$ & $4.2 \mathrm{E}+06$ & 17 \\
\hline TCC & $\mathrm{C} 1$ & $6.8 \mathrm{E}+06$ & $8.2 \mathrm{E}+07$ & $5.0 \mathrm{E}+07$ & $2.8 \mathrm{E}+07$ & 6 \\
[g ${ }^{-1}$ sediment] & $\mathrm{C} 4$ & $1.6 \mathrm{E}+06$ & $4.4 \mathrm{E}+07$ & $1.3 \mathrm{E}+07$ & $1.5 \mathrm{E}+07$ & 6 \\
& $\mathrm{C} 3$ & $3.4 \mathrm{E}+05$ & $3.4 \mathrm{E}+06$ & $1.5 \mathrm{E}+06$ & $1.0 \mathrm{E}+06$ & 6 \\
& $\mathrm{C} 2$ & $1.4 \mathrm{E}+06$ & $4.9 \mathrm{E}+07$ & $1.5 \mathrm{E}+07$ & $1.3 \mathrm{E}+07$ & 17 \\
\hline
\end{tabular}


Table S10: Rank-based Spearman correlation of DNA concentration, bacterial 16S rRNA gene abundance and total cell counts with environmental factors and pore water data. Values in bold are significant $(<0.05)$ when omitting a p-value correction. $\mathrm{R}_{\mathrm{s}^{-}}$values highlighted red show a negative correlation, whereas $r_{s}$-values highlighted green show a positive correlation.

\begin{tabular}{|c|c|c|c|c|c|c|c|c|c|c|c|c|c|c|c|c|c|c|c|c|c|c|c|c|c|c|c|c|}
\hline & $\begin{array}{c}165 \\
\text { Bacteria }\end{array}$ & 16S/DNA & TCC & Temp & Salinity & $\begin{array}{l}\text { Depth } \\
\text { [mbsl] }\end{array}$ & $\begin{array}{l}\text { Depth } \\
\text { [mbs/ } \\
\text { mbsf] }\end{array}$ & $\mathrm{Ba}^{2+}$ & $\mathrm{Ca}^{2+}$ & $\mathrm{K}^{+}$ & $\mathrm{Mg}^{2+}$ & $\mathrm{Na}^{+}$ & $\mathrm{Si}_{\mathrm{aq}}$ & $\mathrm{Cl}$ & $\mathrm{SO}_{4}{ }^{2-}$ & $\mathrm{Br}$ & $\mathrm{NO}_{3}^{-}$ & $\delta 180$ & $\delta \mathrm{D}$ & $\mathrm{pH}$ & TC & TN & TS & Tос & Clay & Silt & Sand & $\begin{array}{c}\text { Grav. } \\
\text { Water } \\
\text { Content }\end{array}$ \\
\hline \multicolumn{29}{|c|}{ p-value } \\
\hline DNA & $>0.001$ & $>0.001$ & $>0.001$ & 0.030 & 0.039 & 0.813 & 0.076 & 0.658 & 0.604 & 0.020 & 0.061 & 0.021 & 0.872 & 0.011 & 0.410 & 0.015 & 0.593 & 0.027 & 0.055 & 0.008 & 0.017 & 0.329 & 0.175 & 0.045 & 0.307 & 0.111 & 0.130 & 0.006 \\
\hline $16 \mathrm{~S}$ Bact. & & $>0.001$ & $>0.001$ & 0.173 & 0.003 & 0.164 & 0.002 & 0.860 & 0.248 & 0.003 & 0.005 & $>0.001$ & 0.475 & $>0.001$ & 0.128 & 0.001 & 0.587 & 0.023 & 0.054 & 0.002 & 0.009 & 0.175 & 0.056 & 0.021 & 0.821 & 0.886 & 0.926 & 0.007 \\
\hline $16 \mathrm{~S} / \mathrm{DNA}$ & & & 0.03 & 0.503 & $>0.001$ & 0.216 & 0.004 & 0.799 & 0.005 & 0.001 & $>0.001$ & $>0.001$ & 0.268 & $>0.001$ & 0.016 & $>0.001$ & 0.425 & 0.369 & 0.528 & 0.001 & 0.218 & 0.171 & 0.135 & 0.284 & 0.055 & 0.102 & 0.084 & 0.153 \\
\hline TCC & & & & $>0.001$ & 0.008 & 0.465 & 0.138 & 0.024 & 0.193 & 0.012 & 0.029 & 0.002 & 0.262 & 0.001 & 0.593 & 0.002 & 0.890 & 0.028 & 0.027 & 0.097 & 0.749 & 0.759 & 0.233 & 0.429 & 0.184 & 0.572 & 0.524 & 0.369 \\
\hline \multicolumn{29}{|c|}{ correlation coefficient $r_{s}$} \\
\hline DNA & 0.87 & 0.47 & 0.68 & -0.37 & -0.35 & 0.04 & 0.30 & -0.08 & -0.09 & -0.39 & -0.32 & -0.39 & -0.03 & -0.43 & -0.14 & -0.41 & 0.09 & -0.37 & -0.33 & -0.44 & 0.40 & 0.17 & -0.23 & 0.34 & 0.18 & 0.27 & -0.26 & 0.47 \\
\hline $16 \mathrm{~S}$ Bact. & & 0.79 & 0.61 & -0.24 & -0.48 & 0.24 & 0.51 & 0.03 & -0.20 & -0.49 & -0.46 & -0.57 & -0.12 & -0.56 & -0.26 & -0.54 & 0.09 & -0.38 & -0.33 & -0.52 & 0.44 & 0.23 & -0.33 & 0.39 & -0.04 & 0.03 & -0.02 & 0.47 \\
\hline $16 \mathrm{~S} / \mathrm{DNA}$ & & & 0.36 & -0.12 & -0.63 & 0.21 & 0.47 & 0.04 & -0.47 & -0.55 & -0.60 & -0.71 & -0.19 & -0.67 & -0.40 & -0.66 & -0.14 & -0.16 & -0.11 & -0.54 & 0.21 & 0.24 & -0.26 & 0.19 & -0.33 & -0.28 & 0.30 & 0.26 \\
\hline TCC & & & & -0.64 & -0.44 & -0.13 & 0.26 & -0.38 & -0.23 & -0.42 & -0.37 & -0.50 & -0.19 & -0.52 & -0.09 & -0.50 & 0.02 & -0.37 & -0.37 & -0.28 & 0.06 & 0.05 & -0.21 & 0.14 & -0.23 & -0.10 & 0.11 & 0.16 \\
\hline
\end{tabular}


Table S11: Significance of the variance introduced by environmental factors into the microbial community tested by Permutational MANOVA (PerMANOVA)

\begin{tabular}{|c|c|c|c|c|}
\hline & $\operatorname{Dim} 1$ & $\operatorname{Dim} 2$ & $r^{2}$ & $p$-value \\
\hline Depth [mbs/msbf] & -0.53174 & -0.84691 & 0.3322 & 0.006 \\
\hline Temperature & 0.13632 & 0.99067 & 0.2487 & 0.015 \\
\hline $\mathrm{Ba}$ & 0.94807 & 0.31805 & 0.1859 & 0.031 \\
\hline $\mathrm{Si}$ & 0.90304 & -0.42956 & 0.1541 & 0.056 \\
\hline $\mathrm{Ca}$ & 0.50032 & 0.86584 & 0.0100 & 0.835 \\
\hline K & 0.81761 & 0.57578 & 0.0612 & 0.341 \\
\hline $\mathrm{Mg}$ & 0.80879 & 0.58809 & 0.0684 & 0.297 \\
\hline $\mathrm{Na}$ & 0.99177 & 0.12804 & 0.0813 & 0.241 \\
\hline Nitrate & -0.81210 & 0.58351 & 0.0280 & 0.637 \\
\hline Chloride & 0.98966 & 0.14344 & 0.0527 & 0.391 \\
\hline Sulfate & -0.28689 & 0.95796 & 0.1014 & 0.161 \\
\hline Bromide & 0.92727 & 0.37439 & 0.0629 & 0.326 \\
\hline Salinity & 0.99532 & 0.09660 & 0.0459 & 0.443 \\
\hline$\delta 180$ & 0.99329 & -0.11569 & 0.3753 & 0.001 \\
\hline$\delta \mathrm{D}$ & 0.98430 & -0.17648 & 0.3914 & 0.001 \\
\hline pH & -0.42785 & 0.90385 & 0.6412 & 0.001 \\
\hline TC & 0.41379 & -0.91037 & 0.1053 & 0.149 \\
\hline TN & -0.38942 & -0.92106 & 0.0268 & 0.640 \\
\hline TS & 0.03653 & 0.99933 & 0.2694 & 0.004 \\
\hline TOC & 0.40692 & -0.91346 & 0.0974 & 0.170 \\
\hline Clay & 0.47503 & 0.87997 & 0.1123 & 0.132 \\
\hline Silt & 0.76336 & 0.64597 & 0.0532 & 0.405 \\
\hline Sand & -0.70792 & -0.70629 & 0.0630 & 0.330 \\
\hline Conductivity & 0.98987 & 0.14199 & 0.0419 & 0.478 \\
\hline
\end{tabular}

Table S12: One-way PerMANOVA of OTU data from each drill site. Summary presents the overall test statistics. Pairwise analysis shows Bonferroni corrected p-values above the diagonal and F-values below.

$\begin{array}{lr}\text { Summary } & \\ \text { Permutation N: } & 9999 \\ \text { Total sum of squares: } & 26.49 \\ \text { Within-group sum of squares: } & 19.41 \\ \text { F: } & 8.276 \\ \text { p (same): } & 0.0001\end{array}$

\begin{tabular}{l|rlll} 
Pairwise & \multicolumn{1}{c}{} & & \\
& \multicolumn{1}{c}{ C1 } & C4 & C3 & C2 \\
\hline C1 & & $\mathbf{0 . 0 0 1 2}$ & $\mathbf{0 . 0 0 0 6}$ & $\mathbf{0 . 0 0 0 6}$ \\
C4 & 4.014 & & $\mathbf{0 . 0 0 0 6}$ & $\mathbf{0 . 0 0 0 6}$ \\
C3 & 12.400 & 7.368 & & $\mathbf{0 . 0 0 0 6}$ \\
C2 & 5.833 & 5.156 & 16.350 &
\end{tabular}


Table S13: Analysis of variance (ANOVA) of DOC concentrations between all four cores and Tukey's pairwise post-hoc test with p-values adjusted according to Copenhaver-Holland above and the Tukey's Q below the diagonal.

$\begin{array}{lrrrrr} & \text { Sum of squares } & \text { df } & \text { Mean square } & \mathbf{F} & \mathbf{p} \text { (same) } \\ \text { Between groups: } & 24714.2 & 3 & 8238.06 & 4.814 & 0.003712 \\ \text { Within groups: } & 155731 & 91 & 1711.34 & \text { Permutation } \mathbf{p}(\mathbf{n}=\mathbf{9 9 9 9 9 )} \\ \text { Total: } & 180446 & 94 & & & 0.02357\end{array}$

\begin{tabular}{c|cccc} 
& C1 & C4 & C3 & C2 \\
\hline C1 & & 0.066 & 0.996 & 0.052 \\
C4 & 3,540 & & 0.299 & $\mathbf{0 . 0 0 2}$ \\
C3 & 0.310 & 2.490 & & 0.739 \\
C2 & 3.676 & 5.209 & 1.441 &
\end{tabular}

Table S14: Fossil bioindicators according to Schirrmeister et al. (2008); Winterfeld et al. (2011); Müller et al. (2009) and their stratigraphical and paleoenvironmental interpretation.

\begin{tabular}{|c|c|c|c|c|c|}
\hline Units & Bioindicator & Stratigraphy & $\begin{array}{l}\text { Landscape, } \\
\text { facies }\end{array}$ & Vegetation & Climate \\
\hline IId & $\begin{array}{l}\text { - Pollen: Cyperaceae, Poaceae, Artemisia, } \\
\text { Salix } \\
\text { - Spores: Encalypta, Glomus } \\
\text { - Green algae: Botryococcus, Pediastrum } \\
\text { - Ostracodes } \\
\text { - Plant macro remains: Carex, Salix sp., } \\
\text { Saxifraga hirculus, Dryas Kobresia } \\
\text { myosuroides, Thlaspitea rotundifolii } \\
\text { - Testacea: hygrophillic (Difflugia), } \\
\text { sphagnobiotic (Heleopera, Nebela, Argynnia } \\
\text { sp.) } \\
\text { - Mammals: Equus caballus, Mammuthus } \\
\text { primigenius }\end{array}$ & $\begin{array}{l}\text { Middle } \\
\text { Weichselian } \\
\text { Interstadial }\end{array}$ & $\begin{array}{l}\text { Floodplain, } \\
\text { alluvial, } \\
\text { boggy, } \\
\text { periodically } \\
\text { flooded }\end{array}$ & $\begin{array}{l}\text { Grass-sedge } \\
\text { tundra }\end{array}$ & $\begin{array}{l}\text { Moderate } \\
\text {, humid }\end{array}$ \\
\hline IIC & No determinable fossil records found & $\begin{array}{l}\text { Early } \\
\text { Weichselian } \\
\text { Stadial }\end{array}$ & fluvial & & \\
\hline $\mathrm{IIb}$ & $\begin{array}{l}\text { Pollen: Larix, Alnus fruticosa, Betula nana, } \\
\text { Ericales }\end{array}$ & $\begin{array}{l}\text { Eemian } \\
\text { Interglacial }\end{array}$ & $\begin{array}{l}\text { Thermokarst } \\
\text { lake }\end{array}$ & Shrub tundra & $\begin{array}{l}\text { Temperat } \\
\text { e }\end{array}$ \\
\hline Ila & No determinable fossil records found & $\begin{array}{l}\text { Early } \\
\text { Weichselian } \\
\text { Stadial }\end{array}$ & fluvial & & \\
\hline I & $\begin{array}{l}\text { - Marine diatoms: Hyalodiscus sp., Paralia } \\
\text { sulcata, Porosira glacialis, Thalassiosira } \\
\text { sp., Thalassiothrix longissima, Centralea }\end{array}$ & Eemian & Thermokarst & Shrub tundra & temperat \\
\hline
\end{tabular}




\begin{tabular}{l|lll}
\hline - & Fresh water diatoms: Naicula radiosa, & Interglacial & lagoon \\
& Eunotia praerupta, Pinnularia gibba, & \\
tetracyclus lacustris & & \\
- Sponge spicula & & \\
- Pollen: Larix, Alnus fruticosa, Betula nana, & \\
& Ericales \\
- Spores: Sphagnum & \\
\hline
\end{tabular}

Herlemann, D. P., Labrenz, M., Jürgens, K., Bertilsson, S., Waniek, J. J. and Andersson, A. F.: Transitions in bacterial communities along the $2000 \mathrm{~km}$ salinity gradient of the Baltic Sea., ISME J., 5(10), 1571-9, doi:10.1038/ismej.2011.41, 2011.

Müller, S., Bobrov, A. A., Schirrmeister, L., Andreev, A. A. and Tarasov, P. E.: Testate amoebae record from the Laptev Sea coast and its implication for the reconstruction of Late Pleistocene and Holocene environments in the Arctic Siberia, Palaeogeogr. Palaeoclimatol. Palaeoecol., 271(3-4), 301-315, doi:10.1016/J.PALAEO.2008.11.003, 2009.

Muyzer, G., De Waal, E. C. and Uitterlinden, A. G.: Profiling of complex microbial populations by denaturing gradient gel electrophoresis analysis of polymerase chain reaction-amplified genes coding for 16S rRNA., Appl. Environ. Microbiol., 59(3), 695-700, 1993.

Schirrmeister, L., Grosse, G., Kunitsky, V., Magens, D., Meyer, H., Dereviagin, A., Kuznetsova, T., Andreev, A., Babiy, O., Kienast, F., Grigoriev, M., Overduin, P. P. and Preusser, F.: Periglacial landscape evolution and environmental changes of Arctic lowland areas for the last 60000 years (western Laptev Sea coast, Cape Mamontov Klyk), Polar Res., 27(2), 249-272, doi:10.1111/j.1751-8369.2008.00067.x, 2008.

Winterfeld, M., Schirrmeister, L., Grigoriev, M. N., Kunitsky, V. V., Andreev, A., Murray, A. and Overduin, P. P.: Coastal permafrost landscape development since the Late Pleistocene in the western Laptev Sea, Siberia, Boreas, 40(4), 697-713, doi:10.1111/j.1502-3885.2011.00203.x, 2011. 